\title{
Understanding the pathogenic evolution of Zika virus
}

\author{
Xiao-Feng Qin ${ }^{1,2}$ \\ ${ }^{1}$ Center of Systems Medicine, Institute of Basic Medical Sciences, Chinese Academy of Medical Sciences and Peking Union Medical College, \\ Beijing 100005, China; \\ ${ }^{2}$ Suzhou Institute of Systems Medicine, Suzhou 215123, China
}

Received April 22, 2016; accepted May 6, 2016; published online May 16, 2016

Citation: Qin, X.F. (2016). Understanding the pathogenic evolution of Zika virus. Sci China Life Sci 59, 737-739. doi: 10.1007/s11427-016-5069-7

Unlike the previous circulating strains that were not associated with significant human pathology, recent circulating Zika virus (ZIKV) strains isolated during the 2015-2016 Brazilian Outbreak are highly suspected to cause neuropathology, including disorders of fetal brain development and Guillain-Barré syndrome (Broutet et al., 2016). Studies have revealed that recent ZIKV strains can be spread through maternal-fetal (Calvet et al., 2016) and sexual (Hills et al., 2016) transmission, in addition to the traditional route of infection via mosquitoes. The unusual characteristics of the recent ZIKV have raised concern globally and necessitated molecular investigation. However, a clear understanding of the molecular evolution and mechanistic basis of increased pathogenicity of the recent ZIKV remains elusive. A study recently published in Cell Host \& Microbe (Wang et al., 2016), by Dr. Genhong Cheng and his colleagues from UCLA in the United States of America and The Chinese Academy of Medical Sciences in China, has taken the first step towards deciphering the pathogenic evolution of ZIKV.

The ZIKV genome is a single-stranded, positive-sense RNA approximately 10,800 bases in length, which encodes three structural $(\mathrm{C}, \mathrm{prM}$, and $\mathrm{E})$ and eight non-structural (NS1, NS2A, NS2B, NS3, NS4A, 2K, NS4B, and NS5) proteins. To ensure complete viral genomic sequencing, a total of $41 \mathrm{ZIKV}$ genomes greater than $10.1 \mathrm{~kb}$ in length were used (Wang et al., 2016). Among these, 28 ZIKV strains were isolated from man, 10 from mosquito, and one

email: fqin1@foxmail.com from monkey. In addition, two newly isolated strains from man, Rio-U1 and Rio-S1, were also included. The phylogenetic tree clearly confirmed two previously known ZIKV lineages, African and Asian, and the Asian lineage origin of the recent ZIKV (Lanciotti and Lambert, 2016). The earliest recorded strain in the Asian lineage, P6-740 (Malaysia/1966), is a mosquito isolate, while all others are human isolates. To further explore the association between evolutionary mutations and the pathology caused by ZIKV, detailed genetic comparisons were performed at three levels, including strains with clinical outcome versus those without, strains from the recent human outbreak versus previous reference human ZIKV, and strains in the Asian versus African lineages (Figure 1).

At the first level, four ZIKV human strains with clear clinical outcomes were analyzed, including Natal_RGN, ZKV2015, Rio-U1, and Rio-S1 (Figure 1). Strains Natal_RGN and ZKV2015 were clearly identified as associated with microcephaly and they were isolated from fetal brain tissue and amniotic fluid from another pregnant patient, respectively. Additionally, the newly reported strains, Rio-U1 and Rio-S1, were not associated with microcephaly; Rio-U1 was isolated from the urine of a pregnant woman, while Rio-S1 was isolated from a man whose illness self-resolved within 10 days. Comparison with 29 other strains of human origin revealed $15,13,16$, and 15 strainspecific nucleotide changes in Natal_RGN, ZKV2015, Rio-U1, and Rio-S1, respectively. Strain-specific amino acid changes in terms of three, three, one, and three mutations, respectively, were also identified. No significant enrichment of these mutations was observed in any specific 
protein. Genetic comparison of ZIKV genomes associated with microcephaly versus those without, will play an important role in the identification of key pathogenic mutations in the future.

At the second level, Wang et al. compared strains from the recent human outbreak with several previously reported reference strains from the Asian lineage including FSM (2007/Mocronesia), H/PF/2013 (French Polyneisa), and P6-740 (1966/Malaysia) (Figure 1). The results of phylogenetic analysis indicated that P6-740 strain isolated from the Aedes aegypti mosquito is the earliest ancestor-like strain of all human isolates from the Asian lineage. Using this as the reference strain, over 400 nucleotide variations and 26 amino acid substitutions were identified in the FSM strain. Furthermore, eight additional conserved amino acid substitutions were found in all recent epidemic strains including FMS, H/PF/2013, and Brazilian strains. Therefore, a total of 34 identical amino acids distributed across prM, E, NS1, NS2A, NS3, NS4B, and NS5 proteins were identified.

At the third level, protein sequences of the strains from the Asian and African lineages were aligned and compared. The results revealed that 59 amino acid variations, which are otherwise conserved across all strains within the Asian or African lineages, are different between the two lineages. Of note, 6 of the 59 variations are enriched in the pr region of prM protein, including I110V, K143E, A148P, V153M, H157Y, and V158I. Moreover, comparison at the second level showed three additional amino acid variations, V123A, S139N, and V153M, located in the pr region of prM protein. The significant enrichment of variations in prM protein indicates its potential important role in the pathogenic evolution of ZIKV. To further investigate the role of these variations, Wang et al. (2016) predicted structural models for pr protein of two representative strains, Rio-U1 from Asian lineage and ARB13565 from African lineage, based on the structure template of dengue type 1 virus. Structural comparisons indicated that A148P variation could cause a 10-amino-acid, structural transformation between a loop and a $\beta$-sheet in pr protein. Taken together, it is tempting to speculate that these well-defined patterns of mutations between ZIKV strains are responsible for the increase in virus infectivity, evasion of maternal immunity to cross the placental barrier, and access to fetal brain tissue.

The reasons for increased pathogenicity of the recent ZIKV outbreak strains, in terms of whether the large naïve host population in the Americas has contributed to the epidemiological difference, are still unclear. However, the report by Wang et al. (2016) clearly opens a new window to investigate the relationship between mutations and viral pathogenicity. Evidently, the stepwise genetic analysis by

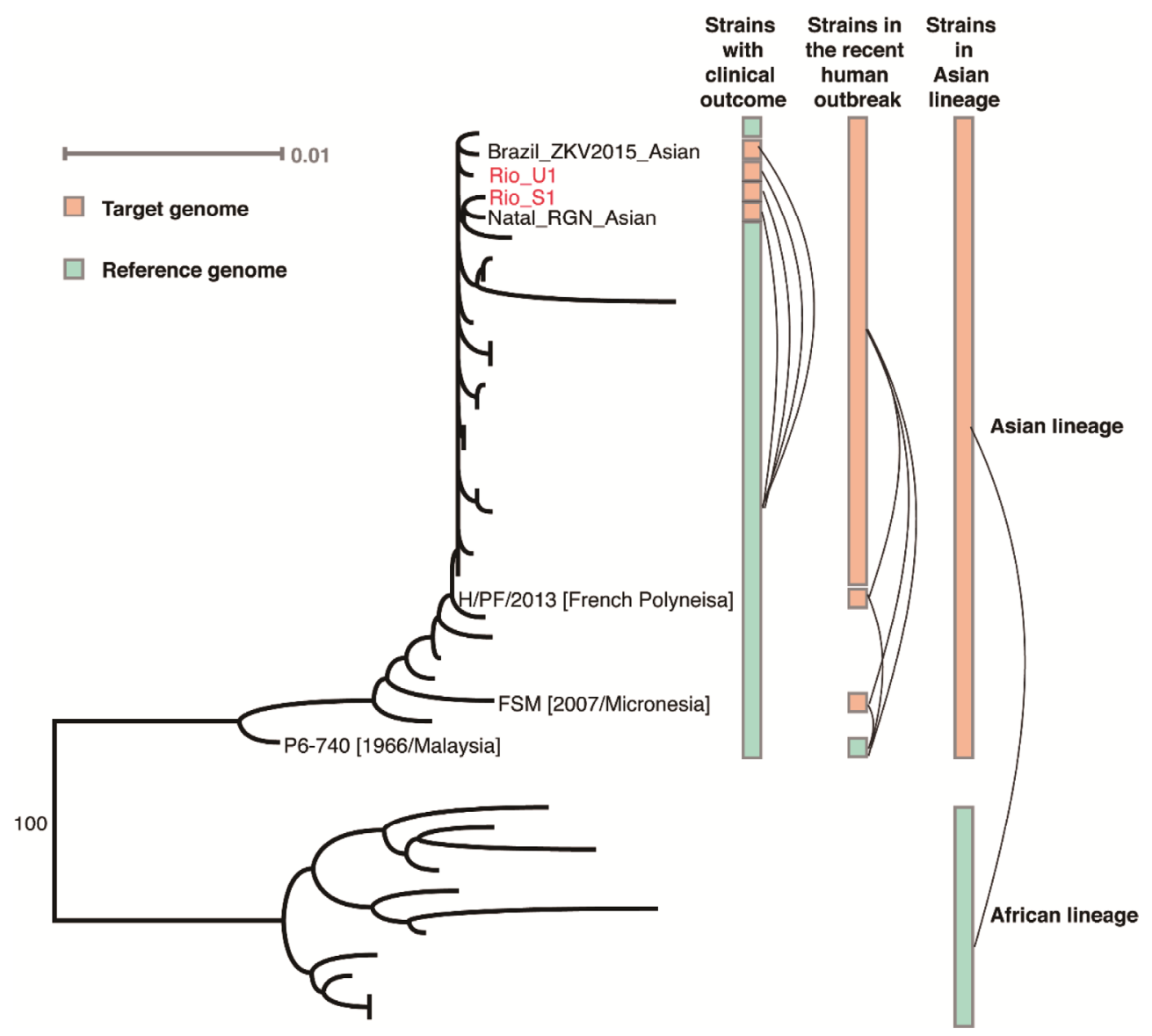

Figure 1 Schematic of phylogenetic and genetic analysis published in Cell Host \& Microbe (Wang et al., 2016). 
Dr. Genhong Cheng and his colleagues has narrowed down the variation candidates that might dominate the viral phenotype. A comprehensive picture is required to better understand the pathogenic evolution of ZIKV.

Compliance and ethics The author(s) declare that they have no conflict of interest.

Broutet, N., Krauer, F., Riesen, M., Khalakdina, A., Almiron, M., Aldighieri, S., Espinal, M., Low, N., and Dye, C. (2016) Zika Virus as a cause of neurologic disorders. N Engl J Med 374, 1506-1509.

Calvet, G., Aguiar, R.S., Melo, A.S., Sampaio, S.A., de Filippis, I., Fabri, A., Araujo, E.S., de Sequeira, P.C., de Mendonça, M.C., de Oliveira,
L., Tschoeke, D.A., Schrago, C.G., Thompson, F.L., Brasil, P., Dos Santos, F.B., Nogueira, R.M., Tanuri, A., and de Filippis, A.M. (2016). Detection and sequencing of Zika virus from amniotic fluid of fetuses with microcephaly in Brazil: a case study. Lancet Infect Dis doi: 10.1016/S1473-3099(16)00095-5.

Hills, S.L., Russell, K., Hennessey, M., Williams, C., Oster, A.M., Fischer, M., and Mead, P. (2016) Transmission of Zika Virus through sexual contact with travelers to areas of ongoing transmission - Continental United States, 2016. MMWR Morb Mortal Wkly Rep 65, 215-216.

Wang, L., Valderramos, S. G., Wu, A., Ouyang, S., Li, C., Brasil, P., Bonaldo, M., Coates, T., Nielsen-Saines, K., Jiang, T., Aliyari, R., and Cheng, G. (2016). From mosquitos to humans: genetic evolution of Zika Virus. Cell Host Microbe doi: 10.1016/j.chom.2016.04.006.

Lanciotti, R.S., and Lambert, A.J. (2016). Phylogenetic analysis of chikungunya virus strains circulating in the western hemisphere. Am J Trop Med Hyg 94, 800-803.

Open Access This article is distributed under the terms of the Creative Commons Attribution License which permits any use, distribution, and reproduction in any medium, provided the original author(s) and source are credited. 